\title{
REVIEW ON AUTOMATIC PROCESSING OF BRAIN IMAGES FOR SEGMENTATION AND ABNORMALITY DETECTION
}

\author{
R. Aishwarya ${ }^{1}$ and Dr. Sumathi Ganesan ${ }^{2}$ \\ ${ }^{1,2}$ Department Of Computer Science And Engineering \\ Annamalai University \\ Annamalai Nagar, Tamil Nadu, India. \\ ${ }^{1}$ aishwaryar.rajendran@gmail.com, ${ }^{2}$ sumi.ganesan@yahoo.com
}

\section{ABSTRACT-}

Brain tumour detection is very popular in the area of medical image processing. This is due to the sensitivity of brain functionality and inter structure. Any kind of ignorance towards the problems related with brain may cause serious impact on human life/life style. Therefore, early detection or diagnosis of abnormalities or tumours helps the doctors and patients to rectify the brain related health problems. The images are obtained through scanning techniques which are very common. Images obtained from the scanning needs to be segmented carefully for the future analysis and damage control procedures. In this paper, a detailed review on different types of segmentation techniques proposed by various authors is studied and compared for a clear understanding of existing segmentation techniques. They are tabulated to summarize different methodologies, segmentation techniques, and existing processes for further studies on Brain image segmentation. Finally, a brief understanding towards deep learning techniques is studied in this paper to understand their role in modern era for automated segmentation process.

Key words and phrases: Segmentation, Tumour, Brain images.

\section{INTRODUCTION}

The brain images are subjected to scrutiny in the medical terms for a detail research and studies to identify the abnormality concerning the patient problems. RAYMOND introduced various MRI techniques. Actually, the author did a research on field-focusing nuclear magnetic resonance (FONAR) for scanning the large image samples by using D.C. magnetic fields [1]. NMR (nuclear magnetic resonance) was focused for safety purpose. In an attempt to find different abnormalities in a brain Hayashi et al. suggested an automated segmentation method for cerebellum and brainstem from the MR images of brain. Using automated segmentation result obtained was around 83 percentage for normal subject matters, 86 percentage arround for the patients. 3D images were used for segmentation and brain. Conventional fuzzy C-mean is used for local spatial context, then during clustering process researchers used intensity nonuniformity (INU) artefact as compensate. In an attempt to emphasize the importance of electrical brain activity Banaschewski and Brandeis suggested 
the usage of electrophysiology towards understanding the brain function in normal conditions or psychiatric conditions of the children or at the time of adolescent age [2]. They used EEG/ERP parameters to understand the brain functioning by monitoring children with different types of brain disorders. Chawla et al. suggested an automated method for detecting and classifying abnormality of acute and chronic infarct along with haemorrhage from the CT images of the brain [3]. To conclude this study, the authors used three steps, i.e. image enhancement, detecting the mid-line symmetry, and finally classifying the abnormal slices. The authors claimed to produce an accuracy of 90 percentage and detection of abnormality as 100 percentage with an average precision of 91percentage. Later, Li et al. created an online database system for the brain images to understand and diagnose subtle brain injuries and tissues that are reflected in the pathological processes [4].

\section{METHODS OF IMAGE SEGMENTATION}

Majorly brain can be segmented in three methods. They are- manual, semiautomatic segmentation and automatic segmentation. Segmentation depends on the complexity of segmenting the brain. Manual segmentation is used when radiologist is very experienced in understanding multimodality and its time consuming process. Semi-automatic segmentation needs user interaction to initialize, intervent and evaluate. Region of interest (ROI) approximates tumour region by initializing in automatic process. Tumour cut method is most used automatic segmentation which needs more attention of radiologist to find tumour region in MRI images. Segmentation had to be applied two times in cellular automata (CA) tumour segmentation. In initial stage tumour seed is provided and in second stage background seed is plotted in probability map.CA algorithm is applied in each MR image to get last outcome of tumour volume. Automatic segmentation is a process of separating normal and abnormal tissues in brain. The changing nature of segmentation process demands the need of obtaining information from different processes like metabolic and psychological using PET, CT and MRI scanning of images There are few other multimodel technologies which combine information obtained from different techniques for obtaining further more accurate tu0mour segmentation process. CT and MRI is used in these technology. The user interaction is completely bypassed in this method and usage of artificial intelligence (AI) and other latest technological tools are driving this method to new levels or towards innovation of new methods.

\section{DIFFERENT METHODS OF AUTOMATIC SEGMENTATION OF BRAIN}

Major automatic segmentation of brain are discussed in detailed information followed by a summarized comparison of the work produced from previous research in this area.

Segmentation of Brain Tumour using MRI Scan images. The MRI scanning is used to diagnose the brain tumour and to treat the patient in an effective way. The special distributions of soft tissues are measured by using T1-relaxation, T2 relaxation and proton density (PD). In the recent time PD images are replaced by fluid attenuated inversion recover (FLAIR) [5]. The Table 1 shows the level of intensity in an MRI scanned image for WM, GM and CSF with H-high, L-low and I-intermediate. A sharp change in image 
function is observed at the edge pixels and this method can be implemented by using Sobel, Roberts, Prewitt and Canny [5] [7]. In fuzzy clustering method every pixel allotted with a defined membership function with a value between 0 to 1 based on their attributes, which represents the similarity between a pixel and its centroids [9]. Artificial neural network consist of three major layer input, output and hidden layer. Processing in this method takes place in hidden layer and output is based on the training in hidden layer [10]. Hybrid method uses pulse-coded NN for stimulation and for segmentation ROI is carried for region growing. PCA reduces dimensionality [5].

TABLE 1. Shows the different levels of intensity for WM, GM, and CSF of an MRI scanned

\begin{tabular}{|c|c|c|c|}
\multicolumn{4}{c}{ images } \\
\hline & T1 & T2 & FLAIR \\
\hline WM & H & L & I \\
\hline GM & I & I & H \\
\hline CSF & L & H & L \\
\hline
\end{tabular}

Segmentation using PET(Positron emission tomography) images. PET gives high accurate over the volume of interest ( VOI). PET is used for treating based on diagnosis and staging. Four types of PET image segmentation methods, Image thresholding which is used for separating tumours with noise background. Vibrantional approach is used if the intensity is varied which gives better sub-pixel at time of segmentation. Stochastic method, is used in identification of neighbouring tissues.

Segmentation of Brain Tumour using CT Images. Segmentation using CT image method helps to provide detailed information with anatomical information and therefore used in radio therapy planning. There are few segmentation method discussed below in Table 2 .

TABLE 2. Methods - Advantages and Disadvantages of Segmentation of MRI brain images

\begin{tabular}{|l|l|l|}
\hline \multicolumn{1}{|c|}{ Method } & \multicolumn{1}{|c|}{ Advantages } & \multicolumn{1}{|c|}{ Disadvantages } \\
\hline $\begin{array}{l}\text { AtlasBased } \\
\text { Segmentation }\end{array}$ & $\begin{array}{l}\text { This method can perform excellent } \\
\text { segmentation and labelling of } \\
\text { variety of regions with a noisy, low } \\
\text { resolution MR images with different } \\
\text { modalities. }\end{array}$ & $\begin{array}{l}\text { Accuracy of the complete method } \\
\text { depends on } \\
\text { topological graph prior's } \\
\text { accuracy. }\end{array}$ \\
\hline $\begin{array}{l}\text { EdgeBased } \\
\text { Segmentation }\end{array}$ & $\begin{array}{l}\text { It is one of the simple methods for } \\
\text { segmentation. }\end{array}$ & $\begin{array}{l}\text { This makes maximum } \\
\text { thickness in boundaries at } \\
\text { the edges. }\end{array}$ \\
\hline GA & $\begin{array}{l}\text { This method has a good selection procedure } \\
\text { for optimal number of regions for the }\end{array}$ & $\begin{array}{l}\text { It has a difficulty to select the } \\
\text { fitness function. }\end{array}$ \\
\hline
\end{tabular}




\begin{tabular}{|l|l|l|}
\hline FCM & $\begin{array}{l}\text { FCM is used for noisy images. } \\
\text { Clustering process is used for reducing } \\
\text { randomness selection process. }\end{array}$ & $\begin{array}{l}\text { Upper and lower approximation } \\
\text { values set up is difficult for } \\
\text { roughness measure. }\end{array}$ \\
\hline $\begin{array}{l}\text { Contour- } \\
\text { Based } \\
\text { Segmentation }\end{array}$ & $\begin{array}{l}\text { MCSS is used with high accuracy in } \\
\text { segmentation process. }\end{array}$ & $\begin{array}{l}\text { Very expensive as compared to } \\
\text { ACM. }\end{array}$ \\
\hline $\begin{array}{l}\text { T1-w } \\
\text { Approach }\end{array}$ & $\begin{array}{l}\text { Used for the brain tissue segmentation over } \\
\text { the images with white lesions. }\end{array}$ & $\begin{array}{l}\text { FLAIR is recommended as } \\
\text { compared with T1-w. }\end{array}$ \\
\hline
\end{tabular}

\section{CHALLENGES INVOLVED IN BRAIN TUMOUR SEGMENTATION}

Segmentation challenge involved in 3D images is detection of gliomas which is from the MRI images. This segmentation includes size, location and shape. This information changes from patient to patient and the variations may have grater difference with the expectations. Edgebased methods are near to impossible due to the unclear boundaries, irregularities and discontinuities in the tumour boundaries. MRI images may have difference in each intensity biases and in each slice from database [6]. To sort out all these challenges multimodel imaging techniques were introduced but they also developed some of the serious challenges like computational complexity, attenuation correction, etc.

\section{CONCLUSIONS}

Automatic segmentation of brain images is considered to be a challenging task due to the involvement of complex techniques and methodologies such as ANN, CNN, etc. Due to the flexibility of accessing various databases from different medical resources the scientists are in a position to understand the existing information from variety of images and methods to provide accurate solutions by segmenting of brain images. This paper reviews major methods which are based on different techniques involved in segmentation of images like MRI, CT and PET images, limitations drawbacks and advantage of brain tumour segmentation of images are discussed. Automated segmentation of brain tumour in clinical application need a lot of improvement by establishing a strong interaction between the clinical researchers and developers.

\section{REFERENCES}

[1] R. DAMADIAN, L. MINKOFF, M. GOLDSMITH, J. A. KOUTCHER : Fieldfocusing nuclear magnetic resonance (FONAR), Nat. 65: (5) 250-252 (1978).

[2] M. CHAWLA, S. SHARMA, J. SIVASWAMY, L. T. KISHORE : A method for automatic detection and classification of stroke from brain CT images, In 2009 Ann. Int. Con. of the IEE. Eng. in Med. and Bio. Soc., IEE. 3581-3584 (2009).

[3] L. LI, D. CHAHAL, J. Z. WANG, M. A. ECKERT, C. LOZAR : Online brain image database system for diagnosis of subtle brain injury, In 2010 4th Int. Con. on Bio. and Bio. Eng., IEE. 1-4 (2010). 
[4] A. IS,IN, C. DIREKOGLU ,M. S,AH : Review of MRI-based brain tumor image segmentation using deep learning methods, Pro. Com. Sci. 102 317-324 (2016).

[5] N. GORDILLO, E. MONTSENY, P. SOBREVILLA : State of the art survey on MRI brain tumor segmentation, Mag. Res. Ima. 31:(8) 1426-1438 (2013).

[6] A. ASLAM, E. KHAN, M. S. BEG : Improved edge detection algorithm for brain tumor segmentation, Pro. Com. Sci. 58 430-437 (2015).

[7] K. SUDHARANI, T. C. SARMA, K. S. PRASAD : Advanced morphological technique for automatic brain tumor detection and evaluation of statistical parameters, Pro. Tec. 24 1374-1387 (2016).

[8] J. V. D. OLIVEIRA, W. PEDRYCZ: Advances in fuzzy clustering and its applications, Eds. Joh. Wil. and Son. (2007).

[9] H. VERMA, R. K. AGRAWAL, A. SHARAN : An improved intuitionistic fuzzy cmeans clustering algorithm incorporating local information for brain image segmentation, App. Sof. Com. 46 543-557 (2016).

[10] M. K. ABD-ELLAH, A. I. AWAD, A. A. KHALAF, H. F. HAMED: A review on brain tumor diagnosis from MRI images: Practical implications, key achievements, and lessons learned, Mag. Res. Ima. (2019).

[11] Somasekar, J. \& Sharma, A. \& Reddy, N. \& Reddy, Y.. (2020). IMAGE ANALYSIS FOR AUTOMATIC ENUMERATION OF RBC INFECTED WITH PLASMODIUM PARASITESIMPLICATIONS FOR MALARIA DIAGNOSIS. Advances in Mathematics: Scientific Journal. 9. 1221-1230. 10.37418/amsj.9.3.48.

[12] A. SHARMA1 AND J. SOMASEKAR "Contrast Image Construction Technique for Medical Imaging" published in Advances in Mathematics: Scientific Journal (Adv. Math., Sci. J.) vol-9no-6-2020 (pp 3325-3329)

[13] Rohini Goel, Avinash Sharma, and Rajiv Kapoor, "Object Recognition Using Deep Learning" published in Journal of Computational and Theoretical Nanoscience Vol. 16, 4044-4052, 2019

[14] Santosh, Mamta \& Sharma, Avinash. (2019). A Proposed Framework for Emotion Recognition Using Canberra Distance Classifier. Journal of Computational and Theoretical Nanoscience. 16. 3778-3782. 10.1166/jctn.2019.8250.

[15] Mamta Santosh, Avinash Sharma, "Facial Expression Recognition using Fusion of LBP and HoG Features" published in International Journal of Innovative Technology and Exploring Engineering (IJITEE) ISSN: 2278-3075, Volume-8 Issue-8 June, 2019 